\title{
Shugoshin: From a perspective of clinical disorders
}

\section{Ravinder Kumar $^{*}$ and Meenakshi Agarwal ${ }^{2}$}

\author{
${ }^{1}$ Department of Obstetrics, Gynecology and Reproductive Sciences, University of California San \\ Francisco, San Francisco-94143, California, USA \\ ${ }^{2}$ Environmental Biotechnology Laboratory, School of the Environment, 1515 S. Martin Luther \\ King Jr. Blvd., FSH Science Research Center, Florida A\&M University, Tallahassee-32307, \\ Florida, USA \\ *Correspondence: Dr Ravinder Kumar, PhD \\ Email: raj86tau@gmail.com \\ Tel.: +1-917-415-5694 (R.K)
}

2

3

Keywords:

Shugoshin; tumor suppressor; cancer; oncogene; tumour associated gene 


\section{ABSTRACT}

33 Proper and timely segregation of cellular genome is an important and a prime requirement of all cell division programmes. Mis-segregation of chromosomes and resulting aneuploidy leads to several clinical consequences. Over the years, shugoshin emerges as a key protein factor involved in the segregation of genetic material in dividing cells. Deletion or altered level of shugoshin is reported in several human malignancies, as a result, shugoshin now emerges as an important tumour associated gene and a possible target for cancer therapy. Apart from the role in cancer, recent studies also showed the involvement of shugoshin in several other clinical disorders. Through this review, we tried to highlight the clinical relevance of shugoshin.

\section{INTRODUCTION}

43 Proper cell division is a foremost requirement for reproduction as well as for the survival and 44 continuity of every species. Mis-segregation of the genome during cell division leads to 45 aneuploidy, which is closely associated with numerous medical consequences ranging from tumorigenesis to sterility, mental retardation, spontaneous abortion, and other birth-related defects

47 [1-6]. To make sure that, the genetic blueprint is duplicated and distributed precisely during cell

48 division, cell employs several mechanisms operating either independently or in coordination with one another. Proper and timely removal of cohesin is an example of one such mechanism. Cohesin, a multiprotein complex holds sister chromatids together since DNA duplication in S-phase till the

51 onset of anaphase. The premature or untimely loss of cohesion as a result of abrupt separase

52 activity leads to chromosome mis-segregation. Hence cohesin cleavage by separase is kept under 53 tight cellular control [7,8]. Apart from its prime role of holding sister chromatids together, cohesin

54 is also known for its involvement in diverse cellular processes discussed elsewhere [9]. The 55 detailed account of cohesin and separase falls outside the scope of the present review and same are 56 summarized elsewhere [8,10]. Apart from the timely cleavage of cohesin, several other

57 mechanisms including DNA damage checkpoint (DDC), spindle assembly checkpoint (SAC), 58 separase activation, centriole duplication (and maybe more which remains unidentified) make sure 59 that the genetic endowment of the cells or organisms i.e. its genome is duplicated and separated 60 properly. The detailed discussion of all such mechanisms is difficult in the present review, may 61 require separate volume and can be found elsewhere [8,11-16]. 
62 In this review, we will mainly focus on the shugoshin, a protein factor required for the protection

63 of centromeric cohesin. The intended purpose of this review is to familiarize readers with the

64 medical conditions in which shugoshin was implicated. Before discussing clinical association of

65 shugoshin, we will gave bird eye view of shugoshin identification, cellular localization, conserved

66 nature, distribution in eukaryotes and finally shugoshin as an emerging tumour associated gene

67 and as a potential target for cancer therapy. The present review does not include description of

68 shugoshin in a cell cycle (mitosis and meiosis) as those topics are already discussed in the recent

69 reviews [17-20].

70 SHUGOSHIN BACKGROUND

71 Shugoshin (for guardian spirit from Japan) is a homo-dimeric phospho-protein belonging to a 72 shugoshin protein family [21,22]. Shugoshin is conserved from single-celled yeast to multicellular 73 mammals including humans. Shugoshin shares several structural features with other members of 74 the shugoshin family including a basic region at the $\mathrm{C}$-terminus which is essential for centromere 75 binding, chromosome localization and an N-terminal coiled-coil domain that may regulate its 76 dimerization and interaction with other proteins $[23,-26]$. Initially, shugoshin was discovered in 77 the fruit fly, D. melanogaster as a peri-centromeric protein (at the time referred as MEI-S332) 78 required for the protection of Rec8 (meiotic-specific cohesin subunit) from separase action and its 79 persistence during meiosis-I [27-29]. Later, a protein factor with a function equivalent to MEI80 S332 was discovered in other eukaryotic species including yeast, insects, vertebrates and plants 81 [30-35].

82 Based on the sequence (at gene and protein level) and structural analysis it is observed that 83 eukaryotic species studied till date possess either one or two genes coding for shugoshin (referred 84 as $S G O 1$ and $S G O 2$ ), although several splicing isoforms of shugoshin have been reported in higher 85 eukaryotes [36]. Table1, shows the number of genes coding for shugoshin in different species. The 86 reason why some species (example Saccharomyces cerevisiae) possess only one gene for 87 shugoshin and others two (example fission yeast, humans) remains elusive. In human cells, a 88 combined total of 10 splicing isoforms (for $S G O 1$ and SGO2) have been identified 89 (http://www.uniprot.org/uniprot/?query=hugoshin\%2C+homo+sapiens\&sort=score). Information 90 related to different isoforms of $\mathrm{SGO1}$ and $\mathrm{SGO} 2$ including number of amino acid residues, 91 molecular mass is given in table 2. Size or number of amino acid residues in shugoshin and 92 molecular mass vary significantly across different eukaryotic species [35] as well as among 
93 different isoforms within a same species (example H. sapiens, table 2). It is important to mention 94 that different shugoshin paralogue is known to exhibit different properties depending on the 95 species under consideration. The expression pattern or profile of shugoshin paralogs may be cell 96 cycle-dependent (i.e., mitosis or meiosis). For example, in fission yeast $S G O 2$ is expressed in both 97 mitosis and meiosis while $S G O 1$ is meiosis-specific [30]. Just like fission yeast, mice $S G O 2$ is 98 required for the completion of meiosis but not for mitosis suggesting cell cycle specific expression 99 [37].

100 Although shugoshin is present in all the eukaryotic species studied till date and shugoshin-based 101 protection of centromeric cohesin is conserved across different eukaryotic species, cells of $C$. 102 elegans use different strategy which is independent of shugoshin. Unlike other species, 103 chromosomes segregation in C. elegans relies on an alternative mechanism which involves $L A B$ 1041 (Long Arm of the Bivalent) [38]. Study from C. elegans raised the possibility of shugoshin 105 independent cohesin protection in other species. Why cells of C. elegans use this alternative 106 mechanism despite the presence of shugoshin remains open question. Whether shugoshin 107 independent protection of centromeric cohesin is exclusive to worms species also remain matter 108 of future investigation.

\section{CELLULAR LOCALIZATION}

110 So far shugoshin has been detected or observed in the nucleus in close association of chromosomes 111 at kinetochore or centromere, at spindle pole body (or SPB, the functional equivalent of centrioles 112 of higher eukaryotes) and more recently at the sub-telomeric region of the chromosome [30,39113 43]. Cellular localization of shugoshin in yeast and the mammalian cell is shown in Figure 1A and 114 1B respectively. By looking at Figure 1, it can be inferred that the overall cellular localization of 115 shugoshin is similar in yeast and mammalian cells despite a huge evolutionary distance. Similar 116 cellular localization of shugoshin in yeast and mammalian cells shows the importance or suitability 117 of yeast as a model system. It is important to mention that in budding yeast, the nuclear membrane 118 always remains intact (closed mitosis) and SPB always remain embedded in the nuclear envelope. 119 In the case of mammalian cells, the nuclear envelope is lost completely (open mitosis), and centrioles always remain in the cytoplasm. Therefore, the possibility of novel shugoshin interactors 121 and role in yeast as well as in mammalian cells cannot be ruled out. Therefore, identification of 122 novel interactors or function represents both opportunities and challenges in front of contemporary 
123 biologist working in this direction. We gave comparative localization of human and yeast cells as 124 great deal of information related to shugoshin is gathered using yeast.

125 SHUGOSHIN AS TUMOUR ASSOCIATED GENE

126 Being an important player in cell cycle, cells make sure that shugoshin is present only when and 127 where needed [44]. Cells also maintain optimum level of shugoshin by regulating its expression 128 and degradation through APC/C (anaphase promoting complex/cyclosome) [45]. Complete 129 absence or altered level (both more and less than the optimum level) of shugoshin may lead to 130 tumorigenesis. Plenty of studies are now available supporting both the oncogenic as well as tumour 131 suppressor nature of shugoshin. Therefore, it will be fair enough to consider shugoshin as a tumour 132 associated gene (Figure 2). Cellular abundance of shugoshin is so critical that even one-fold change 133 in its cellular abundance is enough for tumour induction [46]. The role of shugoshin in cancer is 134 confirmed not only from studies in non-human subjects like rats or mice but also from tissue 135 samples collected from actual cancer patients. Through this section of this review, we highlighted 136 some of the recent studies where complete absence or altered level of shugoshin was associated 137 with cancer.

\section{SHUGOSHIN AS TUMOUR SUPPRESSOR GENE}

139 Decreased level or complete absence of shugoshin was observed in head and neck cancer [47], 140 nasopharyngeal carcinoma [48], neuroblastoma [49], and prostate cancer [50,51]. Homozygous 141 deletion of SGOL2 was observed in different types of human tumours including head and neck 142 cancer [52], small-cell lung carcinoma [53], cervical carcinoma [54], and neuroblastoma [55]. It 143 important to mention that absence or deletion of either shugoshin (i.e., SGOL1 or SGOL2 for 144 shugoshin like in humans) can lead to cancer. Among the 46 colorectal cancer cases, hSgo1 mRNA

145 expression was decreased in the tumour tissue in comparison with the corresponding normal tissue 146 [56]. Heterozygous deletion of sgo1+/- leads to systemic chromosome instability in mice [57] and 147 the formation of ACF (aberrant crypt foci) in mice heterozygous for shugoshin-1 [58]. Treatment 148 with the carcinogen azoxymethane (oxide of azomethane, carcinogenic and neurotoxic chemical 149 compound used in biological research) caused sgo1+/- ME-CIN model mice to develop HCCs 150 (Hepatocellular carcinoma) within 6 months, whereas control mice developed no $\mathrm{HCC}(\mathrm{P}<0.003)$ 151 [59]. These studies showed the tumour suppressor nature of shugoshin.

152 Although $S G O 1+/-$ mice are viable and fertile but showed enhanced colonic tumorigenesis. 153 Enhanced CIN (Cervical intraepithelial neoplasia) observed in sgol-deficient mice leads to an 
154 increase in the formation of an ACF and an accelerated development of tumours on exposure to 155 azoxymethane [60]. The SGOL1-P1 (one of the splicing form of shugoshin in humans) transcript containing an exon-skip of exon 3 that results in a stop codon occurring within exon 4 whose over-

157 expression in human HCT116 cell line resulted in an increased number of cells with aberrant 158 chromosome alignment, precociously separated chromatids and delayed mitotic progression, 159 occasionally followed by inaccurate distribution of the chromosomes [61]. Down-regulation of shugoshin-1 leads to CIN in colorectal cancer cells [56]. Mice heterozygous for shugoshin-1 showed mild proneness to spontaneous lung and liver cancers. In a recent study using adoptive (T/B-cell based) immunity-deficient RAG1(-/-) (Recombination activating gene) sgo1(-/+) double-mutant mice developed lung adenocarcinomas more aggressively compared to sgo1(-/+) or RAG1(-/-) mice, suggesting immune system involvement in CIN-mediated lung carcinogenesis [62]. Up-regulated expression of shugoshin was observed in $82 \%$ of hepatocellular carcinoma (HCC) and correlated with elevated alpha-fetoprotein and early disease onset of HCC while depletion of shugoshin-1 reduced cell viability of hepatoma cell lines including HuH7, HepG2, Hep3B, and HepaRG due to persistent activation of the spindle assembly checkpoint [63]. SHUGOSHIN AS AN ONCOGENE

Increased expression and level of shugoshin was reported in human leukemia [64], and breast cancers [65,66]. Similarly, overexpression of SGOL1-B1 in an NSCLC (Non-small-cell lung carcinoma) cell line induced aberrant chromosome mis-segregation, precociously separated chromatids, and delayed mitotic progression. A Higher level of SGO1-B mRNA was related to taxane [Diterpenes, compounds originally identified in plant genus Taxus (yews), used in cancer chemotherapy e.g., Paclitaxel and docetaxel] resistance, while the forced down-regulation of SGO1-B increased the sensitivity to the taxane [67]. Expression of SGO1C (a non-functional isoform of shugoshin) alone induced aberrant mitosis similar to depletion of $S G O 1 A$, promoting premature sister chromatid separation, activation of the spindle assembly checkpoint, and mitotic arrest suggesting that the expression of $S G O 1 C$ is tightly regulated to prevent dominant-negative effects of $S G O 1 A$ and genome instability [68]. In another clinical study, expression of SGO1 in human prostate tumors were higher than that of adjacent normal tissues and were positively 183 above clearly showed the oncogenic nature of shugoshin. mentioned above clearly showed the oncogenic nature of shugoshin. 
185 Based on the studies mentioned in this section, it can be said that shugoshin can acts as an 186 important target for medical intervention in cancer therapy. Not only complete loss of shugoshin

187 but, the altered level of shugoshin can also lead to cancer. Whether shugoshin association with 188 cancer is due to chromosome mis-segregation or due to derailment of other cellular pathways as a 189 result of complete absence or altered level of shugoshin remains a topic of future research. Since 190 the altered level of shugoshin is associated with diverse cancer, and chemicals [example BPA or 191 Bisphenol A, used as plasticizer in plastic industries] can potentially alter its expression, it might 192 be possible that increased incidences of tumour and associated altered shugoshin level may be 193 linked and need further research [70]. The identification of chemicals that can modulate 194 transcription of shugoshin as well as other tumour associated gene would be an important field of 195 future research.

196 Both complete loss or absence as well as increased expression or increased cellular abundance of 197 shugoshin leads to cancer. Whether oncogenic and tumour suppressor nature of shugoshin 198 modulate same cellular pathways remains unknown.

\section{SHUGOSHIN IN OTHER CLINICAL DISORDERS}

200 In last section we mentioned the tumour associated nature of shugoshin. But cancer or tumour is 201 not only clinical conditions where shugoshin is involved. Research over last several years also 202 implicated shugoshin in other serious medical conditions. Through this section we would like to 203 draw attention towards some of those clinical disorders. Example, recently it was shown that 204 mutations of SGO2 (frameshift, p.Glu485Lysfs*5) and CLDN14 collectively cause coincidental 205 Perrault syndrome which is a rare autosomal genetic disorder characterized by sensorineural 206 hearing loss (SNHL) in males and females and ovarian dysfunction in females [71,72]. Some of 207 the studies in last few years also showed the involvement of shugoshin in neurological disorders 208 like late-onset Alzheimer's disease (LOAD) [73,74]. It is important to mention that not all the 209 diseased conditions are due to chromosome instability, which is induced by impaired shugoshin 210 function of centromeric cohesion. For example, Chronic Atrial and Intestinal Dysrhythmia (CAID) 211 is not due to chromosome instability due to impaired shugoshin at the centromere [75,76].

212 FINAL CONCLUSIONS AND FUTURE DIRECTIONS

213 Based on the results from different scientific groups working on a various model system and tissue 214 samples from cancer patients, it can be clearly said that shugoshin is an important tumour 215 associated gene. Apart from role in cancer, shugoshin is also involved in several other medical 
216 conditions. It important to mention that shugoshin associated pathies may also happen even when

217 there is no observed chromosomal instability and shugoshin may still be involved. The way

218 shugoshin is associated with different clinical conditions, it may be possible the list of shugoshin

219 associated pathies may increase in the future. In such a scenario, the availability of high-resolution

220 atomic structure of shugoshin will help in designing or screening for small molecules with

221 applications in cancer therapy and other diseases. Apart from this, availability of structure will

222 help in better understanding shugoshin localization and its interaction with other cellular proteins

223 or complexes. The availability of high-resolution structure will also be important in better

224 understanding the way shugoshin is involved in different diseases. The diverse and big size of

225 shugoshin pose a big challenge for structure biologist [24,77]. Apart from this, innately disordered

226 regions in most shugoshin proteins make it difficult to get a high-resolution structure. The presence

227 of different splicing isoforms of shugoshin in higher eukaryotes poses another hurdle in structural

228 studies. Furthermore, we are still not aware of the cellular abundance of shugoshin, how it is

229 regulated, and what protein factors and signaling is involved in maintaining the optimum level of

230 shugoshin in a cell. Therefore, future research on shugoshin will be important and rewarding from

231 point of basic science as well as from prospective of medical science.

233 Author Contributions: RK conceive the idea, started writing the draft and prepare the figures and 234 tables. MA contributed to writing the draft and manages the reference section.

235 Funding: The author declares that no funding to be reported.

236 Acknowledgments: We regret not being able to refer to the work of everyone in the field. We are

237 thankful to the University of California San Francisco for providing space and other necessary

238 facilities which helped us in completing this manuscript.

239 Conflicts of Interest: The author declares that no conflict of interest exists.

240 Data Availability Statement: Data sharing does not apply to this article, as no new data were 241 created or analyzed in this study.

242 ORCID ID: Dr. Ravinder Kumar, PhD: https://orcid.org/0000-0003-1922-0030 


\section{REFERENCES}

248 1. Rajagopalan, H., Lengauer, C. Aneuploidy and cancer. Nature 2004,432, 338-341.

249 2. Hassold, T., Hall, H., Hunt, P. The origin of human aneuploidy: where we have been, where 250 we are going. Hum. Mol. Genet. 2007,16, 203-208.

251 3. Jallepalli, P.V., Lengauer, C. Chromosome segregation and cancer: Cutting through the 252 mystery. Nat Rev Cancer 2001,1, 109-117.

253 4. Lengauer, C., Kinzler, K.W., Vogelstein, B. Genetic instability in colorectal cancers. 254 Nature 1997,386, 623-627.

255 5. Griffin, D.K. The incidence, origin, and etiology of aneuploidy. Int Rev Cytol. 1996, 167, 256 263-296.

257 6. Sen, S. Aneuploidy and cancer. Curr Opin Oncol. 2000, 12, 82-88.

258 7. Uhlmann, F. Secured cutting: Controlling separase at the metaphase to anaphase transition. 259 EMBO Rep. 2001, 2, 487-492.

2608 8umar, R. Separase: Function Beyond Cohesion Cleavage and an Emerging Oncogene. J 261 Cell Biochem 2017, 118, 1283-1299.

262 9. Mehta, G.D., Kumar, R., Srivastava, S., Ghosh, S.K. Cohesin: functions beyond sister 263 chromatid cohesion. FEBS Lett. 2013, 587, 2299-312.

264 10. Nasmyth, K., Haering, C.H. Cohesin: Its roles and mechanisms. Annu Rev Genet. 2009, $26543,525-558$.

266 11. Joglekar, A.P. A Cell Biological Perspective on Past, Present and Future Investigations of 267 the Spindle Assembly Checkpoint. Biology (Basel), 2016, 5, pii: E44.

268 12. Kamenz, J., Hauf, S. Time To Split Up: Dynamics of Chromosome Separation. Trends Cell 269 Biol. 2017, 27, 42-54.

270 13. Fujita, H., Yoshino, Y., Chiba, N. Regulation of the centrosome cycle. Mol Cell Oncol. $2712015,3 \mathrm{e} 1075643$.

272 14. Simpson-Lavy, K.J., Oren, Y.S., Feine, O., Sajman, J., Listovsky, T., Brandeis, M. Fifteen 273 years of APC/cyclosome: a short and impressive biography. Biochem Soc Trans. 2010, 38, 78-82.

274 15. Agarwal, M., Mehta, G., and Ghosh SK. Role of Ctf3 and COMA subcomplexes in 275 meiosis: Implication in maintaining Cse4 at the centromere and numeric spindle poles. Biochimica 276 et Biophysica Acta-Molecular Cell Research 2015, 1853(3),671-684 
277 16. Agarwal, M., Jin, H., McClain, M., Fan, J., Koch, B.A., Jaspersen, S.L. and Yu, H.G. The 278 half-bridge component Kar1 promotes centrosome separation and duplication during budding 279 yeast meiosis. Molecular Biology of the cell 2018, 29, 1798-1810.

280 17. Marston, A.L. Shugoshins: tension-sensitive pericentromeric adaptors safeguarding 281 chromosome segregation. Mol Cell Biol. 2015, 35, 634-48.

282 18. Clift, D., Marston, A.L. The role of shugoshin in meiotic chromosome segregation. 283 Cytogenet Genome Res. 2011, 133, 234-42

284 19. Wassmann, K. Sister chromatid segregation in meiosis II: deprotection through 285 phosphorylation. Cell Cycle 2013, 12, 1352-9

286 20. Zhang, Q., Liu, H. Functioning mechanisms of Shugoshin-1 in centromeric cohe-sion 287 during mitosis. Essays Biochem. 2020, 64, 289-297.

288 21. Xu, Z., Cetin, B., Anger, M., Cho, U. S., Helmhart, W., Nasmyth, K., Xu, W. Structure and 289 function of the PP2A-shugoshin inter-action. Molecular Cell 2009, 35, 426-441.

290 22. Swaney, D.L., Beltrao, P., Starita, L., Guo, A., Rush, J., Fields, S., Krogan, N.J., Villén, J. 291 Global analysis of phosphorylation and ubiquitylation crosstalk in protein degradation. Nat 292 Methods 2013, 10, 676-682.

293 23. Tang, T.T., Bickel, S.E., Young, L.M., Orr-Weaver, T.L. Maintenance of sister-chromatid 294 cohesion at the centromere by the Drosophila MEI-S332 protein. Genes Dev. 1998, 12, 3843-3856.

295 24. Watanabe, Y. Shugoshin: guardian spirit at the centromere. Current Opinion in Cell 296 Biology 2005, 17, 590-595.

297 25. Watanabe, Y., Kitajima, T.S. Shugoshin protects cohesin complexes at centromeres. Philos 298 Trans R Soc Lond B Biol Sci. 2005, 360, 515-21.

299 26. Watanabe, Y. Temporal and spatial regulation of targeting aurora B to the inner 300 centromere. Cold Spring Harb Symp Quant Biol. 2010, 75, 419-423.

301 27. Davis, B.K. A analysis of a meiotic mutant resulting in precocious sister-centromere 302 separation in Drosophila melanogaster. Mol Gen Genet. 1971, 113, 251-272.

303 28. Kerrebrock, A.W., Miyazaki, W.Y., Birnby, D., Orr-Weaver, T.L. The Drosophila mei304 S332 gene promotes sister-chromatid cohesion in meiosis following kinetochore differentiation. 305 Genetics 1992, 130, 827-841. 
306 29. Kerrebrock, A., Moore, D., Wu, J., Orr-Weaver, T. Mei-S332, a Drosophila protein 307 required for sister-chromatid cohesion, can localize to meiotic centromere regions. Cell 1995, 83, 308 247-256.

309 30. Kitajima, T.S., Kawashima, S.A., Watanabe, Y. The conserved kinetochore protein 310 shugoshin protects centromeric cohesion during meiosis. Nature 2004, 427, 510-517

311 31. Katis, V.L., Galova, M., Rabitsch, K.P., Gregan, J., Nasmyth, K. Maintenance of cohesin 312 at centromeres after meiosis I in bud-ding yeast requires a kinetochore-associated protein related 313 to MEI-S332. Curr Biol. 2004, 14, 560-572.

314 32. Marston, A.L., Tham, W.H., Shah, H., Amon, A. A genome-wide screen identifies genes 315 required for centromeric cohesion. Science 2004, 303, 1367-1370.

316 33. Rabitsch, K.P., Gregan, J., Schleiffer, A., Javerzat, J.P, Eisenhaber, F., Nasmyth, K. Two 317 fission yeast homologs of Drosophila Mei-S332 are required for chromosome segregation during 318 meiosis-I and II. Curr Biol. 2004, 14, 287-301.

319 34. Hamant, O., Golubovskaya, I., Meeley, R., Fiume, E., Timofejeva, L., Schleiffer, A., 320 Nasmyth, K., Cande, W.Z. A REC8-dependent plant Shugoshin is required for maintenance of 321 centromeric cohesion during meiosis and has no mitotic functions. Curr Biol. 2005, 15, 948-54.

322 35. Wang, M., Tang, D., Wang, K., Shen, Y., Qin, B., Miao, C., Li, M., Cheng, Z. OsSGO1 323 maintains synaptonemal complex stabili-zation in addition to protecting centromeric cohesion 324 during rice meiosis. Plant J. 2011, 67, 583-594.

325 36. Yao, Y., Dai, W. Shugoshins function as a guardian for chromosomal stability in nuclear 326 division. Cell Cycle 2012, 11, 2631-2642.

327 37. Llano, E., Gómez, R., Gutiérrez-Caballero, C., et al. Shugoshin-2 is essential for the 328 completion of meiosis but not for mitotic cell division in mice. Genes Dev. 2008, 22, 2400-2413.

329 38. de Carvalho, C.E., Zaaijer, S., Smolikov, S., Gu, Y., Schumacher, J.M, Colaiácovo, M.P. 330 LAB-1 antagonizes the Aurora B kinase in C. elegans. Genes Dev. 2008, 22, 2869-2885.

331 39. Huh, W.K., Falvo, J.V., Gerke, L.C., Carroll, A.S., Howson, R.W., Weissman, J.S., 332 O'Shea, E.K. Global analysis of protein local-ization in budding yeast. Nature 2003, 425, 686-91. 333 40. Kiburz, B.M., Reynolds, D.B., Megee, P.C., Marston, A.L., Lee, B.H., Lee, T.I., Levine, 334 S.S., Young, R.A., Amon, A. The core cen-tromere and Sgol establish a 50-kb cohesin-protected 335 domain around centromeres during meiosis I. Genes Dev 2005, 19, 3017-30. 
336 41. Mishra, P.K., Thapa, K.S., Chen, P., Wang, S., Hazbun, T.R., Basrai, M.A. Budding yeast 337 CENP-ACse4 interacts with the N-terminus of Sgo1 and regulates its association with centromeric 338 chromatin. Cell Cycle 2018, 17, 11-23.

339 42. Tashiro, S., Handa, T., Matsuda, A., Ban, T., Takigawa, T., Miyasato, K., Ishii, K., Kugou, 340 K., Ohta, K., Hiraoka, Y., Masukata, H., Kanoh, J. Shugoshin forms a specialized chromatin 341 domain at subtelomeres that regulates transcription and replication timing. Nat Commun. 2016, 7, 34210393.

343 43. Kanoh, J. Unexpected roles of a shugoshin protein at subtelomeres. Genes Genet Syst. $3442018,92,127-133$.

345 44. McGuinness, B.E., Hirota, T., Kudo, N.R., Peters, J.M., Nasmyth, K. Shugoshin prevents 346 dissociation of cohesin from centro-meres during mitosis in vertebrate cells. PLoS Biol. 2005, 3, 347 e86.

348 45. Karamysheva, Z., Diaz-Martinez, L.A., Crow, S.E., Li, B., Yu, H. Multiple anaphase349 promoting complex/cyclosome degrons mediate the degradation of human Sgo1. J Biol Chem. $3502009,284,1772-1780$.

351 46. Mu J, Fan L, Liu D, Zhu D. Overexpression of shugoshin1 predicts a poor prognosis for 352 prostate cancer and promotes metas-tasis by affecting epithelial-mesenchymal transition. Onco 353 Targets Ther. 2019,12,1111-1118.

354 47. Coon, S.W., Savera, A.T., Zarbo, R.J., Benninger, M.S., Chase, G.A., Rybicki, B.A., \& 355 Van Dyke, D.L. Prognostic implications of loss of heterozygosity at 8p21 and 9p21 in head and 356 neck squamous cell carcinoma. Int. J. Cancer 2004, 111, 206-212.

357 48. Shao, J.Y., Wang, H.Y., Huang, X.M., Feng, Q.S., Huang, P., Feng, B.J., Huang, L.X., Yu, 358 X.J., Li, J.T., Hu, L.F., Ernberg, I., Zeng, Y.X. Genome-wide allele type analysis of sporadic 359 primary nasopharyngeal carcinoma from southern China. Int. J. Oncol. 2000, 17, 1267- 1275.

360 49. Altura, R.A., Maris, J.M., Li, H., Boyett, J.M., Brodeur, G.M., Look, A.T. Novel regions 361 of chromosomal loss in familial neu-roblastoma by comparative genomic hybridization. Genes 362 Chromosomes Cancer 1997, 19, 176-184.

363 50. Pallai, R., Bhaskar, A., Barnett-Bernodat, N., Gallo-Ebert, C., Nickels, J.T. Jr., Rice, L.M. 364 Cancerous inhibitor of protein phos-phatase 2A promotes premature chromosome segregation and 365 aneuploidy in prostate cancer cells through association with shugoshin. Tumour Biol. 2015,36, 366 6067-6074. 
367 51. Dahiya, R., McCarville, J., Hu, W., Lee, C., Chui, R.M., Kaur, G., Deng, G. Chromosome 368 3p24- 26 and 3p22-12 loss in human prostatic adenocarcinoma. Int. J. Cancer 1997, 71, 20-25.

369 52. Beder, L.B., Gunduz, M., Ouchida, M., Fukushima, K., Gunduz, E., Ito, S., Sakai, A., 370 Nagai, N., Nishizaki, K., Shimizu, K. Ge-nome-wide analyses on loss of heterozygosity in head 371 and neck squamous cell carcinomas. Lab. Invest. 2003, 83, 99-105.

372 53. Kohno, T., Morishita, K., Takano, H., Shapiro, D.N., Yokota, J. Homozygous deletion at 373 chromosome 2 q33 in human small-cell lung carcinoma identified by arbitrarily primed PCR 374 genomic fingerprinting. Oncogene 1994, 9, 103-108.

375 54. Rader, J.S., Kamarasova, T., Huettner, P.C., Li, L., Li, Y., Gerhard, D.S. Allelotyping of 376 all chromosomal arms in invasive cer-vical cancer. Oncogene 1996, 13, 2737-2741.

377 55. Takita, J., Yang, H.W., Chen, Y.Y., Hanada, R., Yamamoto, K., Teitz, T., Kidd, V., 378 Hayashi, Y. Allelic imbalance on chromo-some $2 \mathrm{q}$ and alterations of the caspase 8 gene in 379 neuroblastoma. Oncogene 2001, 20, 4424- 4432.

380 56. Iwaizumi, M., Shinmura, K., Mori, H., Yamada, H., Suzuki, M., Kitayama, Y., Igarashi, 381 H., Nakamura, T., Suzuki, H., Watanabe, Y., Hishida, A., Ikuma, M., Sugimura, H. Human Sgo1 382 downregulation leads to chromosomal instability in colo-rectal cancer. Gut 2009, 58, 249-260.

383 57. Rao, C.V., Sanghera, S., Zhang, Y., Biddick, L., Reddy, A., Lightfoot, S., Janakiram, N.B., 384 Mohammed, A., Dai, W., Yamada, H.Y. Systemic Chromosome Instability Resulted in Colonic 385 Transcriptomic Changes in Metabolic, Proliferation, and Stem Cell Regulators in Sgo1-/+ Mice. 386 Cancer Research 2016, 76, 630-642

387 58. Rao, C.V., Sanghera, S., Zhang, Y., Biddick, L., Reddy, A., Lightfoot, S., Dai, W., 388 Yamada, H.Y. Antagonizing pathways lead-ing to differential dynamics in colon carcinogenesis 389 in Shugoshin1 (Sgo1)-haploinsufficient chromosome instability model. Mol Carcinogenesis 2016, $39055,600-610$.

391 59. Yamada, H.Y., Zhang, Y., Reddy, A., Mohammed, A., Lightfoot, S., Dai, W., Rao, C.V. 392 Tumor-promoting/progressing role of additional chromosome instability in hepatic carcinogenesis 393 in Sgol (Shugoshin 1) haploinsufficient mice. Carcinogenesis 2015, 36, 429-440.

394 60. Yamada, H.Y, Yao, Y., Wang, X., Zhang, Y., Huang, Y., Dai, W., Rao, C.V. 395 Haploinsufficiency of SGO1 results in deregulated centrosome dynamics, enhanced chromosomal 396 instability and colon tumorigenesis. Cell Cycle 2012, 11, 479-488. 
397 61. Kahyo, T., Iwaizumi, M., Shinmura, K., Matsuura, S., Nakamura, T., Watanabe, Y., 398 Yamada, H., Sugimura, H. A novel tu-mor-derived SGOL1 variant causes abnormal mitosis and 399 unstable chromatid cohesion. Oncogene 2011, 30, 4453-63.

400 62. Yamada, H.Y., Kumar, G., Zhang, Y., Rubin, E., Lightfoot, S., Dai, W., Rao, C.V. 401 Systemic chromosome instability in Shugosh-in-1 mice resulted in compromised glutathione 402 pathway, activation of Wnt signaling and defects in immune system in the lung. Oncogenesis 2016, $4035, \mathrm{e} 256$.

404 63. Wang, L.H., Yen, C.J., Li, T.N., Elowe, S., Wang, W.C., Wang, L.H. Sgo1 is a potential 405 therapeutic target for hepatocellular car-cinoma. Oncotarget 2015, 6, 2023-2033.

406 64. Yang J, Ikezoe T, Nishioka C, Yokoyama, A. A novel treatment strategy targeting 407 shugoshin 1 in hematological malignancies. Leuk Res. 2013, 37, 76-82.

408 65. Scanlan, M.J., Gout, I., Gordon, C.M., Williamson, B., Stocker, Et., Gure, A.O., Jager, D., 409 Chen, Y.T., Mackay, A., O’Hare, M.J., Old, L.J. Humoral immunity to human breast cancer: 410 antigen definition and quantitative analysis of mRNA expression. Can-cer Immun. 2001, 1, 4.

411 66. Yang, Q., Yoshimura, G., Nakamura, M., Nakamura, Y., Shan, L., Suzuma, T., Tamaki, 412 T., Umemura, T., Mori, I., Kakudo, K. Allelic loss of chromosome 3p24 correlates with tumor 413 progression rather than with retinoic acid receptor beta2 expression in breast carcinoma. Breast 414 Cancer Res. Treat. 2011, 70, 39- 45.

415 67. Matsuura, S., Kahyo, T., Shinmura, K., Iwaizumi, M., Yamada, H., Funai, K., Kobayashi, 416 J., Tanahashi, M., Niwa, H., Ogawa, H., Takahashi, T., Inui, N., Suda, T., Chida, K., Watanabe, 417 Y., Sugimura, H. SGOL1 variant B induces abnormal mitosis and resistance to taxane in non418 small cell lung cancers. Scientific Reports 2013, 3, 3012.

419 68. Wong, W.K., Kelly, T., Li, J., Ma, H.T., Poon, R.Y. SGO1C is a non-functional isoform 420 of Shugoshin and can disrupt sister chromatid cohesion by interacting with PP2A-B56. Cell Cycle $4212015,14,3965-3977$.

422 69. Chen Q, Wan X, Chen Y, Liu C, Gu M, Wang Z. SGO1 induces proliferation and 423 metastasis of prostate cancer through AKT-mediated signaling pathway. Am J Cancer Res. $4242019,9,2693-2705$.

425 70. Ribeiro-Varandas, E., Viegas, W., Sofia Pereira, H., Delgado, M. Bisphenol A at 426 concentrations found in human serum in-duces aneugenic effects in endothelial cells. Mutat Res. 427 2013, 751, 27-33. 
428 71. Domínguez-Ruiz, M., García-Martínez, A., Corral-Juan, M. et al. Perrault syndrome with 429 neurological features in a com-pound heterozygote for two TWNK mutations: overlap of TWNK430 related recessive disorders. J Transl Med 2019, 17, 290.

431 72. Faridi, R., Rehman, A.U., Morell, R.J., Friedman, P.L., Demain, L., Zahra, S., Khan, A.A., 432 Tohlob, D., Assir, M.Z., Beaman, G., Khan, S.N., Newman, W.G., Riazuddin, S., Friedman, T.B. 433 Mutations of SGO2 and CLDN14 collectively cause coincidental Perrault syndrome. Clin Genet. $4342017,91,328-332$.

435 73. Rao, C.V., Farooqui, M., Zhang, Y., Asch, A.S., Yamada, H.Y. Spontaneous development 436 of Alzheimer's disease-associated brain pathology in a Shugoshin-1 mouse cohesinopathy model. 437 Aging Cell 2018, 17,e12797.

438 74. Rao CV, Farooqui M, Madhavaram A, Zhang Y, Asch AS, Yamada HY. GSK3439 ARC/Arg3.1 and GSK3-Wnt signaling axes trigger amyloid- $\beta$ accumulation and 440 neuroinflammation in middle-aged Shugoshin 1 mice. Aging Cell 2020,19,e13221.

441 75. Zhang, Q., Liu, H. Functioning mechanisms of Shugoshin-1 in centromeric cohesion 442 during mitosis. Essays Biochem. 2020, 64, 289-297.

443 76. Chetaille, P., Preuss, C., Burkhard, S. et al. Mutations in SGOL1 cause a novel 444 cohesinopathy affecting heart and gut rhythm. Nat Genet 2014, 46, 1245-1249.

445 77. Wang, X., Dai, W. Shugoshin, a guardian for sister chromatid segregation. Exp Cell Res. $4462005,310,1-9$. 


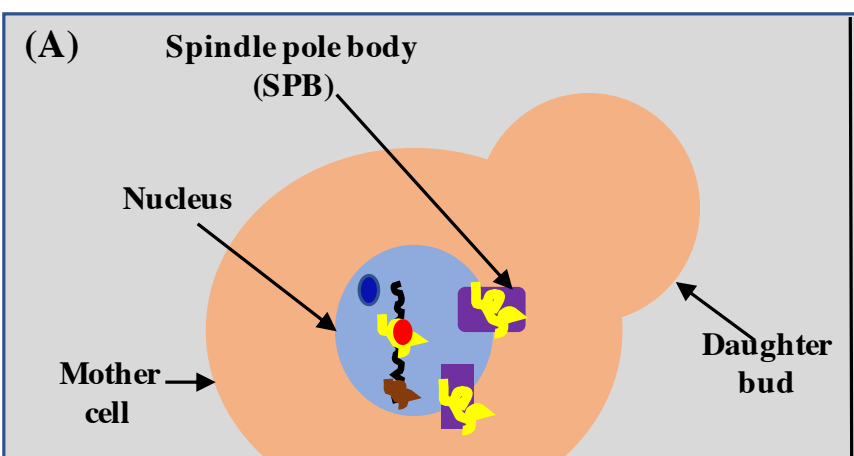

Yeast cell
(B)

B)

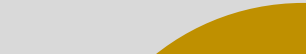

Nucleus

$x$

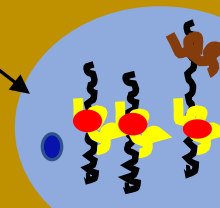

Cell membrane

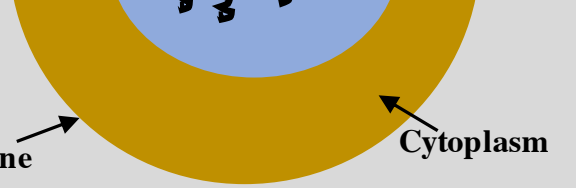

Mammalian cell

Bound Shugoshin $>$ Sub-telomeric shugoshin

Unbound shugoshin in nucleus Centromere

Figure 1. Cellular localization of shugoshin. Localization of shugoshin in (A) budding yeast and (B) mammalian cell. Note: Diagrams are for demonstration purpose only.

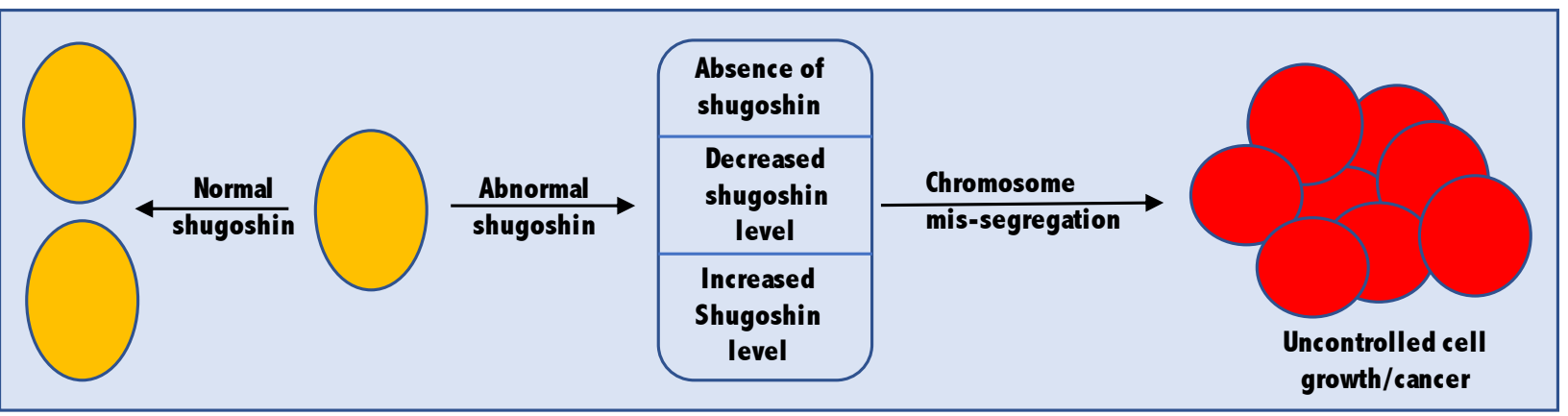

Figure 2. Shugoshin as tumour associated gene. Complete absence as well as increased or decreased level of shugoshin was found to be associated with different type of cancers. 
480 Table 1. Number of genes coding for shugoshin in different species.

\begin{tabular}{cccc}
\hline Species & No. of Genes & Kingdom & References \\
\hline Saccharomyces cerevisiae & 1 & Fungi & $*$ \\
Schizosaccharomyces pombe & 2 & Fungi & $*$ \\
Mus masculus & 2 & Animalia & Uniprot \\
Arabidopsis thialana & 2 & Plantae & Uniprot \\
sHomo sapiens & 2 & Animalia & Uniprot \\
Drosophila melanogaster & 1 & Animalia & Uniprot \\
Caenorhabditis elegence & 1 & Animalia & Uniprot \\
Oryza sativa & 1 & Plantae & Uniprot \\
Xenopus laevis & 1 & Animalia & Uniprot \\
Neurospora crassa & 1 & Fungi & Uniprot \\
Danio rerio & 1 & Animalia & Uniprot \\
Zea mays & 1 & Plantae & Uniprot \\
Rattus norvegicus & 1 & Animalia & Uniprot \\
Candida glabrata & 1 & Fungi & $*$ \\
Kluyveromyces lactis & 1 & Fungi & $*$ \\
Aphis gossypii & 1 & Fungi & $*$ \\
Pristionchus pacificus & 1 & Animalia & Uniprot \\
Oryzias latipes & 1 & Animalia & Uniprot \\
Candida albicans & 1 & Fungi & $*$ \\
\hline
\end{tabular}

481

482

483

484

485

486

*https://portals.broadinstitute.org/cgibin/regev/orthogroups/show_orthogroup.cgi?orf=YOR073W.

Uniprot (www.uniprot.org/uniprot).

$\S$ Total ten isoforms have been reported in humans.

Table 2. Size, molecular mass of different isoforms of human SGO1 and SGO2

\begin{tabular}{lllll} 
Shugoshin & Isoform & $\begin{array}{l}\text { Number of amino } \\
\text { acid residues }\end{array}$ & $\begin{array}{l}\text { Mol. Mass } \\
\text { (in kDa) }\end{array}$ & Identifier \\
\hline${ }^{*}$ SGO1 & Isoform 1 & 561 & 64.19 & Q5FBB7-1 \\
& Isoform 2 & 309 & 35.344 & Q5FBB7-2 \\
& Isoform 3 & 292 & 33.501 & Q5FBB7-3 \\
& Isoform 4 & 275 & 31.276 & Q5FBB7-4 \\
& Isoform 5 & 258 & 29.433 & Q5FBB7-5 \\
& Isoform 6 & 527 & 60.122 & Q5FBB7-6 \\
& Isoform 7 & 215 & 24.646 & Q5FBB7-7 \\
& Isoform 1 & 1265 & 144.739 & Q562F6-1 \\
& Isoform 2 & 1261 & 144.181 & Q562F6-2 \\
& Isoform 3 & 247 & 28.23 & Q562F6-3 \\
\hline
\end{tabular}

*https://www.uniprot.org/uniprot/Q5FBB7

! https://www.uniprot.org/uniprot/Q562F6 